\title{
An overview of RNA virus-encoded microRNAs
}

\author{
Xihan Li and Xiaoping Zou
}

\begin{abstract}
MicroRNAs (miRNAs) are a number of small non-coding RNAs playing a regulatory part in gene expression. Many virus-encoded miRNAs have been found, which manifests that viruses as well apply the basic pattern of gene regulation, however, mostly in viruses transcribed from double-stranded DNA genomes. It is still in dispute if RNA viruses could encode miRNAs because the excision of miRNA might result in the cleavage of viral RNA genome. We will focus on the miRNAs encoded by RNA virus and discuss their potential role in viral replication cycle and host cells.
\end{abstract}

\section{Discovery and biogenesis of microRNAs}

MicroRNAs (miRNAs) are a number of small noncoding RNAs which typically silence the expression of genes via various mechanisms [1-3]. MiRNAs are key factors in regulating gene expression of various cellular processes, so the discovery of miRNAs turns out to be a noteworthy breakthrough of molecular biology [4-8]. In 1993, there had been researchers observed MiRNA lin-4 in the Caenorhabditis elegans [2, 8]. In subsequent researches, other similar small regulatory RNAs were discovered in various organisms. It has been illustrated that the small RNA originated from a hairpin structure which are partly complementary to the 3 'untranslated regions (UTR) of other target transcripts [9]. MRNA destabilization and translational repression can be triggered by this binding, resulting in protein production decline $[10,11]$. MiRNAs are estimated to have an influence on $60 \%$ of mammalian gene expression [12]. Recent studies indicate that miRNAs produce main effect in various regulatory pathways, for instance metabolism, apoptosis, proliferation and differentiation of cells, embryonic development, cancer, and so on $[13,14]$.

In mammals, miRNAs are created in a multi-step process. The biogenesis pathway of miRNA has been studied in detail. Canonical miRNAs derived from hairpin-shaped transcripts (pri-miRNAs) which are usually transcribed by RNA polymerase II (pol II) [15].

* Correspondence: 13770771661@163.com

Department of Gastroenterology, Affiliated Drum Tower Hospital, Nanjing University School of Medicine, Nanjing, Jiangsu, China
Then the precursor miRNAs (pre-miRNAs) are cleaved out of the larger pri-miRNA by the RNAse III-like endonuclease Drosha[16]. The pri-miRNA is conducted via the nuclear microprocessor complex, which comprises the double-stranded RNA (dsRNA)-discerning DiGeorge-syndrome critical-region protein 8 (DGCR8) and endonuclease Drosha [17]. Then the hairpin pre-miRNA is carried out of the nucleus by the nuclear transport receptor, Exportin-5 and finally to the cytoplasm [18, 19]. Then in the presence of RNAse III-like endonuclease Dicer, the pre-miRNAs are cleaved after entering into the cytoplasm [20]. A short duplex RNA is generated by dicer-mediated cleavage. Over the RNA-induced silencing complex (RISC) activation process, one strand of the duplex called miRNA, remains stably combined into the complex (RISC*) and undertakes as a sequence-specific probe targeting RISC* to the complementary mRNA [21]. Another strands, is released, degraded and partly complementary target sequences [22].

\section{Virus-encoded microRNAs}

As intracellular pathogens, viruses are related to a lot of diseases in plants and animals. Normally viruses use the biosynthetic molecular mechanism to multiply in host cell. Not surprisingly, viruses can generate miRNAs in their own genomes as well, which may generally take advantage of the host gene expression. Shortly after the first miRNA was identified, the first virus-encoded miRNAs was discovered for the human Epstein-Barr virus (EBV) [23]. Up to now, more than 250 novel viral miRNAs were discovered, which provides the possibility 
to explore the function and biogenesis of virus-encoded miRNAs [24]. Although the function of miRNAs in DNA virus has been described in detail, yet if RNA viruses could encode miRNAs is less understood. A possible explanation is that most RNA virus duplicates in cytoplasm and the viral RNAs could not interact with the nuclear microprocessor complex mediating the biosynthesis of miRNA $[25,26]$. Another reason is the processing of miRNAs from the viral genome will destroy the viral RNA then impact the replication of virus $[25,27]$. Viruses, however, are able to alter cellular pathways out of their self-interest, several researches employed ultra-sensitive mechanisms to discover miRNAs encoded by RNA virus $[28,29]$. We describe the features of miRNAs encoded by RNA virus as well as their underlying biological function involved in gene regulation. An over-view of miRNAs encoded by RNA viruses is given in Table 1 .

\section{MicroRNAs encoded by retroviruses}

Retroviruses are a unique class of enveloped small RNA viruses replicating via reverse transcription and integrating the dsDNA copy of their own genome into the host's genome [27]. As a result, retroviruses are potential RNA viruses to generate miRNAs, because all the retroviral transcription originates from host machinery which is similar with the directing expression of miRNAs in cells.

Researchers have studied whether retroviruses like human immunodeficiency virus-1 (HIV-1) may encode miRNAs [30]. It was reported that the (TAR) motif was the source of some miRNAs encoded by HIV [31, 32]. TAR is a stable hairpin structure resembling miRNA precursors and it is necessary for the activation of HIV-1 transcription [33]. Several researchers described
TAR-derived miRNAs in latently infected and productively human cells, and via chromatin remodeling, the TAR-derived miRNAs can start up transcriptional silencing at the long terminal repeats (LTR) promoter, even downregulate apoptotic genes [34]. In another research using MT-4 T cells infected HIV-1, a new miRNA designated miR-N367, was separated within the nef region of the viral genome, and a role in downregulating both nef function and HIV-1 transcription by the LTR U3 region negative-response element [35]. Kaul et al. [36] illustrated that HIV1-miR-H1, a miRNA encoded by HIV-1, represses the cellular miRNA miR-149 of host which aims the viral accessory protein named Vpr. It is demonstrated that the Pol and Env protein-coding regions of the HIV-1 genome generate a few sequences like miRNA which are homologous with human miR-30e, miR-195, miR-424 and miR-374a [35, 37, 38].

B-cell tumors naturally developed in cattle are associated with the infection of bovine leukemia virus (BLV), a retrovirus with RNA genome [39]. It is reported that BLV generates a conserved cluster of miRNAs [40]. Interestingly, different from other known miRNAs, this kind of miRNAs are not cleaved by the endonuclease Drosha, but shorter RNA polymerase III (pol III), only the subgenomic small RNAs are cleaved into miRNAs [41]. This permits the mRNA transcripts and viral genome to avoid cleavage. The BLV miRNAs are extremely expressed in malignant and leukemic cells where the gene expressions of virus are inhibited, proposing a function on tumor progression and onset [42]. BLV-miR-B4, which is one of BLV miRNAs, has same targets with miRNA miR-29 of the host [40]. It is illustrated that both bovine miR-29a and BLV-miR-B4 target two transcripts related to B-cell tumorigenesis induced

Table 1 MiRNAs encoded by RNA viruses

\begin{tabular}{|c|c|c|c|c|}
\hline Virus & MiRNA & Targets & Proposed function & Reference \\
\hline \multirow[t]{2}{*}{ HIV-1 } & miR-N367, & LTR & Downregulate Nef function and virulence & {$[35,38]$} \\
\hline & HIV1-miR-H1 & AATF & Initiates mononuclear cells apoptosis & {$[36]$} \\
\hline Bovine leukemia virus & BLV-miR-B4 & HBP1, PXDN & $\begin{array}{l}\text { Mimics miR-29 and contributing to BLV } \\
\text { induced tumorigenesis }\end{array}$ & {$[40,41]$} \\
\hline Bovine foamy virus & $\begin{array}{l}\text { BF2-3p } \\
\text { miR-BF1-5p } \\
\text { miR-BF1-3p } \\
\text { miR-BF2-5p, miR- }\end{array}$ & & & [43] \\
\hline Avian leukosis virus & $E(X S R)$ & & & [45] \\
\hline West Nile virus & KUN-miR-1 & GATA4 & Facilitates virus replication & {$[48]$} \\
\hline Dengue virus & DENV-vsRNA-5 & NS1 & Virus autoregulation & [52] \\
\hline H5N1 influenza virus & miR-HA-3p & PCBP2 & $\begin{array}{l}\text { Contributing to 'cytokine storm' } \\
\text { and mortality }\end{array}$ & [54] \\
\hline Ebola virus & $\begin{array}{l}\text { miR-VP-3p } \\
\text { EBOV-miR-1-5p } \\
\text { EBOV-miR-1-3p } \\
\text { EBOV-miR-2-3p }\end{array}$ & & & {$[56-58]$} \\
\hline
\end{tabular}


by miR-29 in mice, this discoveries put forward a potential mechanism that contributes to BLV-induced tumorigenesis.

It was recently reported that bovine foamy virus (BFV) could generate miRNAs out of a Pol III transcript within both cattle and cultured cells infected by BFV [43]. In this research, a single pri-miRNA (122-nt long) subsequently cleaves into two pre-miRNAs then transformed into miRNAs. The transcript of the miRNA gene consists in both 30 and 50 end of the integrated pregenome, because the pri-miRNA is generated in the U3 region of LTR.

Recently, an alpharetrovirus named avian leukosis virus (ALV) was discovered to generate a miRNA in the exogenous virus-specific region, named XSR or E element [44]. The E-XSR element exists in some ALV and Rous Sarcoma Virus (RSV) strains, and their oncogenicity has been illustrated [45]. Unlike the BFV and BLV, ALV appears to utilize the typical miRNA biogenesis pathway, encodes the miRNA via RNA Pol II and depends on Dicer and Drosha in the processes.

\section{MicroRNA-like small RNA encoded by West Nile virus (WNV)}

West Nile virus (WNV), a single-chain and positive-sense RNA virus, causes possibly deadly diseases infecting animals and humans over the world [46]. The 3'-UTR, especially the terminal 3' stem -loop (3'SL) achieves multiple roles in virus-host interactions and virus replication [47]. Mazhar et al. [48] discovered this highly conserved 3'SL generates a precursor of a 21-nt small viral regulatory RNA (svrRNA) and encodes a microRNA-like small RNA, named KUN-miR-1. What's more, silence of Dicer-1 but not Dicer-2 in Aag2 cells leads to a prominent reduction of KUN-miR-1 levels and WNV virus replication. In-depth study identifies KUNmiR-1 targets the cellular mosquito GATA4 mRNA which results in up-regulation of KUNmiR-1in cells and GATA4-induction using KUN-miR-1 plays a crucial part in the process of virus replication of WNV in mosquito cells. Nevertheless, the small RNA KUNmiR-1is not produced in mammalian cells infected by WNV. It is speculated that extra regulatory elements in mammalian cells may prevent the conversion of SL to mature miRNA. Another possible reason, KUNmiR-1 may be encoded not much in mammalian cells then consequently not detected by the experimental conditions applied.

\section{MicroRNA-like small RNA encoded by dengue virus (DENV)}

Dengue virus (DENV), a single-stranded RNA virus, leads to widespread mortal diseases affecting many people all over the world $[49,50]$. There are four different serotypes of DENV, includes DENV-1, DENV-2,
DENV-3, and DENV-4 [51]. Mazhar et al. [52] demonstrates the production of a functional microRNA-like viral small RNA (vsRNA) encoded by DENV-2. Further research about six vsRNAs derived DENV demonstrates the suppression of DENV-vsRNA-5 may cause obvious increases in the replication of DENV. Besides, experiments illustrate that DENV-vsRNA-5 targets the nonstructural protein 1 (NS1) sequences of DENV and as a result regulates viral genome negatively.

\section{MicroRNA-like small RNA encoded by H5N1 influenza virus}

$\mathrm{H} 5 \mathrm{~N} 1$ influenza virus, a single-stranded and negative-sense RNA, leads to the highest mortality in all influenza viruses [53]. It is reported that $\mathrm{H} 5 \mathrm{~N} 1$ influenza virus generates a microRNA-like small RNA, which was named miR-HA-3p. MiR-HA-3p is produced out of a stem loop-including viral RNA precursor cleaved by Ago 2 using Solexa sequencing, qRT-PCR assays and northern blot [54]. Further research demonstrates that the suppression of poly ( $\mathrm{rC}$ )-binding protein 2 (PCBP2), which is an negative regulator of RIG-I/MAVS regulated by miR-HA-3p could induce 'cytokine storm' in H5N1 virus infected macrophages of mice and human. This discovery provides a possibly efficient treatment strategy to deal with H5N1 infection, which is based on antagomir-HA-3p.

\section{MicroRNA-like fragment encoded by Ebola virus (EBOV)}

EBOV is a negative-strand RNA virus which duplicates in the cytoplasm and leads to a severe hemorrhagic fever [55]. It is reported that EBOV can encode miRNA-like fragment to destroy host immune defenses [56, 57]. Chen et al. [58] speculates three pre-miRNAs by the EBOV/Yambuku-Mayinga sequence and keeps one pre-miRNA after alignment with 125 EBOV genomes, then this pre-miRNA creates one mature miRNA sequence, miR-VP-3p. Further research discovers the miRNA-like fragment exists in serum of Ebola virus disease (EVD) patients by Northern blotting, qRT-PCR and TA-cloning/sequencing. Interesting, subsequent consequences discover that this miRNA-like fragment exists during the acute phase but not during recovery phase in the serum of EBOV-positive patients. With great clinical importance, this miRNA-like fragment is detectable before the detection of Ebola genomic RNA, which may improve the diagnosis of EVD.

\section{Perspective}

Gene expression of virus might be influenced by cellular miRNAs, and gene expression of cell and virus might be influenced by viral miRNAs. MiRNAs encoded by DNA viruses are properly demonstrated. The possibility of 
DNA viruses to encode miRNAs is less surprising because DNA viruses have giant genome size and are capable of duplicating in nucleus and associate with a lot of host proteins [25]. While whether RNA viruses express miRNAs in a homothetic way to host miRNAs continues to be in dispute. In the past few years, several publications have put forward the capability of RNA viruses to encode miRNAs. The function of miRNAs in pathogenesis and replication of RNA virus begins to come up. Even if what we realize with respect to miRNAs and RNA viruses is stirring, it's quite limited and requires further exploration. In the near future, studies will not only enhance our total comprehension of RNA virus-encoded miRNA, but also supply critical informations about the evolution of miRNA-mediated adjustment of infection caused by RNA virus and potentially new insights of therapeutic relevance.

\section{Abbreviations}

ALV: Avian leukosis virus; BFV: Bovine foamy virus; BLV: Bovine leukemia virus; DENV: Dengue virus; DGCR8: DiGeorge-syndrome critical-region protein 8; DNA: Deoxyribonucleic acid; dsRNA: double-stranded ribonucleic acid; EBOV: Ebola virus; EBV: Epstein-Barr virus; HIV: Human immunodeficiency virus; LTR: the long terminal repeats; miRNA: micro ribonucleic acid; NS1: Nonstructural protein 1; PCBP2: Poly ( $\mathrm{rC}$ )-binding protein 2; Pol II: RNA polymerase II; Pre RNA: precursor ribonucleic acid; RISC: RNA-induced silencing complex; RNA: micro ribonucleic acid; RSV: Rous Sarcoma Vir: svrRNA: small viral regulatory ribonucleic acid; TAR: Transactivation responsive ribonucleic acid; UTR: Untranslated region; WNV: West Nile virus; XSR: exogenous virus-specific region

\section{Acknowledgements}

We thank Dr. Ying Huang (Affiliated Drum Tower Hospital, Medical School of Nanjing University) for critical reading of the manuscript.

\section{Funding}

This work was supported by grants from the National Natural Science Foundation of China (No. 31500125), the Natural Science Foundation of Jiangsu Province (No. BK20171124), the Jiangsu Provincial Commission of Health and Family Planning (No. H2017049), and the Science and Technology Program of Nanjing (No. 201803037).

\section{Availability of data and materials}

Not applicable.

\section{Authors' contributions}

$\mathrm{XL}$ wrote the manuscript, $\mathrm{XZ}$ revised the manuscript. All authors read and approved the final manuscript.

\section{Ethics approval and consent to participate}

Not applicable.

\section{Consent for publication}

Not applicable.

\section{Competing interests}

The authors declare that they have no competing interests.

\section{Publisher's Note}

Springer Nature remains neutral with regard to jurisdictional claims in published maps and institutional affiliations.
Received: 6 September 2018 Accepted: 18 March 2019

Published online: 08 October 2019

\section{References}

1. Lagos-Quintana M, Rauhut R, Lendeckel W, et al. Identification of novel genes coding for small expressed RNAs. Science. 2001;294(5543):853-8.

2. Lau NC, Lim LP, Weinstein EG, et al. An abundant class of tiny RNAs with probable regulatory roles in Caenorhabditis elegans. Science. 2001; 294(5543):858-62.

3. Lee RC, Ambros V. An extensive class of small RNAs in Caenorhabditis elegans. Science. 2001;294(5543):862-4.

4. Dooley J, Linterman MA, Liston A. MicroRNA regulation of T-cell development. Immunol Rev. 2013;253(1):53-64.

5. Guedes J, Cardoso AL, Pedroso DLM. Involvement of microRNA in microgliamediated immune response. Clin Dev Immunol. 2013;2013:186872.

6. Lamouille S, Subramanyam D, Blelloch R, et al. Regulation of epithelialmesenchymal and mesenchymal-epithelial transitions by microRNAs. Curr Opin Cell Biol. 2013;25(2):200-7.

7. Ponomarev ED, Veremeyko T, Weiner HL. MicroRNAs are universal regulators of differentiation, activation, and polarization of microglia and macrophages in normal and diseased CNS. Glia. 2013;61(1):91-103.

8. Lee RC, Feinbaum RL, Ambros V. The C. elegans heterochronic gene lin-4 encodes small RNAs with antisense complementarity to lin-14. Cell. 1993; 75(5):843-54.

9. Reinhart BJ, Slack FJ, Basson M, et al. The 21-nucleotide let-7 RNA regulates developmental timing in Caenorhabditis elegans. Nature. 2000;403(6772): 901-6.

10. Guo H, Ingolia NT, Weissman JS, et al. Mammalian microRNAs predominantly act to decrease target mRNA levels. Nature. 2010;466(7308): 835-40.

11. Baek D, Villen J, Shin C, et al. The impact of microRNAs on protein output. Nature. 2008;455(7209):64-71.

12. Chen K, Rajewsky N. The evolution of gene regulation by transcription factors and microRNAs. Nat Rev Genet. 2007:8(2):93-103.

13. Alvarez-Garcia I, Miska EA. MicroRNA functions in animal development and human disease. Development. 2005;132(21):4653-62.

14. Ambros $V$. The functions of animal microRNAs. Nature. 2004:431(7006):350-5.

15. Bartel DP. MicroRNAs: genomics, biogenesis, mechanism, and function. Cell. 2004;116(2):281-97.

16. Slezak-Prochazka I, Durmus S, Kroesen BJ, et al. MicroRNAs, macrocontrol: regulation of miRNA processing. RNA. 2010;16(6):1087-95.

17. Carthew RW, Sontheimer EJ. Origins and mechanisms of miRNAs and siRNAs. Cell. 2009;136(4):642-55.

18. Lee $Y$, Ahn C, Han J, et al. The nuclear RNase III Drosha initiates microRNA processing. Nature. 2003;425(6956):415-9.

19. Zeng Y, Yi R, Cullen BR. Recognition and cleavage of primary microRNA precursors by the nuclear processing enzyme Drosha. EMBO J. 2005; 24(1):138-48.

20. Kim VN. MicroRNA biogenesis: coordinated cropping and dicing. Nat Rev Mol Cell Biol. 2005:6(5):376-85.

21. Grimson A, Farh KK, Johnston WK, et al. MicroRNA targeting specificity in mammals: determinants beyond seed pairing. Mol Cell. 2007;27(1):91-105

22. Bartel DP. MicroRNAs: target recognition and regulatory functions. Cell. 2009;136(2):215-33.

23. Pfeffer S, Zavolan M, Grasser FA, et al. Identification of virus-encoded microRNAs. Science. 2004;304(5671):734-6.

24. Qi P, Han JX, Lu YQ, et al. Virus-encoded microRNAs: future therapeutic targets? Cell Mol Immunol. 2006;3(6):411-9.

25. Harwig A, Das AT, Berkhout B. Retroviral microRNAs. Curr Opin Virol. 2014;7: $47-54$.

26. Cullen BR. Five questions about viruses and microRNAs. PLoS Pathog. 2010; 6(2):e1000787.

27. Houzet $L$, Jeang KT. MicroRNAs and human retroviruses. Biochim Biophys Acta. 2011;1809(11-12):686-93.

28. Lin J, Cullen BR. Analysis of the interaction of primate retroviruses with the human RNA interference machinery. J Virol. 2007;81(22):12218-26.

29. Umbach $J$, Yen $H L$, Poon $L L$, et al. Influenza A virus expresses high levels of an unusual class of small viral leader RNAs in infected cells. mBio. 2010;1(4): e00204-10.

30. Bennasser Y, Le SY, Yeung ML, et al. HIV-1 encoded candidate micro-RNAs and their cellular targets. Retrovirology. 2004;1:43. 
31. Zhang Y, Fan M, Geng G, et al. A novel HIV-1-encoded microRNA enhances its viral replication by targeting the TATA box region. Retrovirology. 2014;11(1):23.

32. Berkhout B. Structural features in TAR RNA of human and simian immunodeficiency viruses: a phylogenetic analysis. Nucleic Acids Res. 1992; 20(1):27-31.

33. Klaver B, Berkhout B. Evolution of a disrupted TAR RNA hairpin structure in the HIV-1 virus. EMBO J. 1994;13(11):2650-9.

34. Berkhout B, Silverman RH, Jeang KT. Tat trans-activates the human immunodeficiency virus through a nascent RNA target. Cell. 1989;59(2):273-82.

35. Omoto S, Fujii YR. Regulation of human immunodeficiency virus 1 transcription by nef microRNA. J Gen Virol. 2005;86(Pt 3):751-5.

36. Kaul D, Ahlawat A, Gupta SD. HIV-1 genome-encoded hiv1-mir-H1 impairs cellular responses to infection. Mol Cell Biochem. 2009;323(1-2):143-8.

37. Bennasser $Y$, Le SY, Benkirane $M$, et al. Evidence that HIV-1 encodes an siRNA and a suppressor of RNA silencing. Immunity. 2005;22(5):607-19.

38. Omoto $S$, Ito M, Tsutsumi $Y$, et al. HIV-1 nef suppression by virally encoded microRNA. Retrovirology. 2004;1:44

39. Gillet N, Florins A, Boxus M, et al. Mechanisms of leukemogenesis induced by bovine leukemia virus: prospects for novel anti-retroviral therapies in human. Retrovirology. 2007:4:18.

40. Kincaid RP, Burke JM, Sullivan CS. RNA virus microRNA that mimics a B-cell oncomiR. Proc Natl Acad Sci U S A. 2012;109(8):3077-82.

41. Rosewick N, Momont M, Durkin K, et al. Deep sequencing reveals abundant noncanonical retroviral microRNAs in B-cell leukemia/lymphoma. Proc Natl Acad Sci U S A. 2013;110(6):2306-11.

42. Kettmann R, Cleuter $Y$, Gregoire D, et al. Role of the $3^{\prime}$ long open reading frame region of bovine leukemia virus in the maintenance of cell transformation. J Virol. 1985;54(3):899-901.

43. Whisnant AW, Kehl T, Bao Q, et al. Identification of novel, highly expressed retroviral microRNAs in cells infected by bovine foamy virus. J Virol. 2014; 88(9):4679-86

44. Chen M, Granger AJ, Vanbrocklin MW, et al. Inhibition of avian leukosis virus replication by vector-based RNA interference. Virology. 2007;365(2):464-72.

45. Yao $Y$, Smith $L P$, Nair $V$, et al. An avian retrovirus uses canonical expression and processing mechanisms to generate viral microRNA. J Virol. 2014;88(1):2-9.

46. Mackenzie JS, Gubler DJ, Petersen LR. Emerging flaviviruses: the spread and resurgence of Japanese encephalitis, West Nile and dengue viruses. Nat Med. 2004;10(12 Suppl):S98-S109.

47. Davis WG, Blackwell JL, Shi PY, et al. Interaction between the cellular protein eEF1A and the 3'-terminal stem-loop of West Nile virus genomic RNA facilitates viral minus-strand RNA synthesis. J Virol. 2007;81(18):10172-87.

48. Hussain M, Torres S, Schnettler E, et al. West Nile virus encodes a microRNAlike small RNA in the $3^{\prime}$ untranslated region which up-regulates GATA4 mRNA and facilitates virus replication in mosquito cells. Nucleic Acids Res. 2012:40(5):2210-23.

49. Gubler DJ. Epidemic dengue/dengue hemorrhagic fever as a public health, social and economic problem in the 21st century. Trends Microbiol. 2002; 10(2):100-3.

50. Scott TW, Morrison AC. Vector dynamics and transmission of dengue virus: implications for dengue surveillance and prevention strategies: vector dynamics and dengue prevention. Curr Top Microbiol Immunol. 2010;338:115-28.

51. Alvarez DE, De Lella EA, Fucito $S$, et al. Role of RNA structures present at the $3^{\prime} U T R$ of dengue virus on translation, RNA synthesis, and viral replication. Virology. 2005;339(2):200-12.

52. Hussain M, Asgari S. MicroRNA-like viral small RNA from dengue virus 2 autoregulates its replication in mosquito cells. Proc Natl Acad Sci U S A. 2014;111(7):2746-51.

53. Korteweg C, Gu J. Pathology, molecular biology, and pathogenesis of avian influenza a (H5N1) infection in humans. Am J Pathol. 2008:172(5):1155-70.

54. Li X, Fu Z, Liang H, et al. H5N1 influenza virus-specific miRNA-like small RNA increases cytokine production and mouse mortality via targeting poly (rC)binding protein 2. Cell Res. 2018;28(2):157-71.

55. Nouvellet $\mathrm{P}$, Garske T, Mills HL, et al. The role of rapid diagnostics in managing Ebola epidemics. Nature. 2015;528(7580):S109-16.

56. Liang $\mathrm{H}$, Zhou Z, Zhang $\mathrm{S}$, et al. Identification of Ebola virus microRNAs and their putative pathological function. Sci China Life Sci. 2014;57(10):973-81.

57. Duy J, Honko AN, Altamura LA, et al. Virus-encoded miRNAs in Ebola virus disease. Sci Rep. 2018;8(1):6480.

58. Chen Z, Liang H, Chen X, et al. An Ebola virus-encoded microRNA-like fragment serves as a biomarker for early diagnosis of Ebola virus disease. Cell Res. 2016;26(3):380-3.

\section{Ready to submit your research? Choose BMC and benefit from:}

- fast, convenient online submission

- thorough peer review by experienced researchers in your field

- rapid publication on acceptance

- support for research data, including large and complex data types

- gold Open Access which fosters wider collaboration and increased citations

- maximum visibility for your research: over $100 \mathrm{M}$ website views per year

At $\mathrm{BMC}$, research is always in progress.

Learn more biomedcentral.com/submissions 